\title{
EXPERIMENTAL STUDIES ON THE MOISTURE- DEPENDENT SPREAD OF INJECTION AGENTS
}

\author{
Astrid WALTER* \\ Faculty of Engineering and Information Technology, University of Pécs \\ Boszorkány u. 2, H-7624 Pécs, Hungary, e-mail: awalter@mnet-mail.de
}

Received 31 December 2017; accepted 5 September 2018

\begin{abstract}
Additional horizontal sealing in mineral building materials often produce insufficient or varying results despite the application of the injection agent being carried out according to the manufacturers' specifications.

There is no current scientific explanation for this effect, which prompted this research. The main idea was to solve the question of whether the increasing filling of the capillary pores of mineral building materials with water (and therefore the degree of moisture penetration) can be connected to the varying spread of the injection agent.

After developing a test procedure, different degrees of moisture penetration were applied to samples of bricks and mortar cubes and hydrophobic injection agents were applied to the test specimens. The results showed that the degree of spreading of the injection agent was inversely proportional to the degree of moisture penetration. Consequently there is a dependence of the above mentioned parameters (the existing degree of moisture penetration and the spreading of the injection agent) and a model was developed of the spread of injection agents dependent on the existing degree of moisture penetration in building materials.
\end{abstract}

Keywords: Injection agent, Subsequent horizontal sealing, Bricks, Mortar, model of spread of injection agents

\section{Introduction}

Damage to mostly historical buildings caused by moisture is still a worldwide problem. Additional horizontal sealing can be carried out as one of several possible

\footnotetext{
${ }^{*}$ Corresponding Author
} 
remedies, but often the results are less than adequate. On this topic the author had already published (e.g. [1], [2]), so this article is to be seen as a continuation of considerations in building physics.

The main reasons for this are:

- Until now there are no legal standards how to realize a functional horizontal sealing and therefore the results vary greatly;

- There are no standardized methods of testing the results and consequently no comparability of the results;

- Often no preliminary investigations of an injection agent are done before application. This carries the risk of a wrong choice of injection agent or the inappropriate method of the sealing itself;

- Frequently there is an overestimation of the effectiveness of an injection agent as a result of misleading instructions from the manufacturer ('this agent can be used up to $100 \%$ of the existing moisture degree');

- 'Application by chance', i.e. this time the application of the sealing is successful, the other time the application is a failure although the application was carried out according to the manufacturers specifications. The reason lies in the fact that there is no general transferability of important parameters as e.g. the borehole distance, as well as there being no consideration of the existing moisture degree of the masonry.

These facts caused the leading question of what the reasons for the obviously insufficient spreading of the injection agent are and therefore the varying results of a subsequent horizontal sealing.

Among other research activities, dealing with the computer-aided evaluation of transport mechanisms of salt and water in building materials [3], [4], the following considerations of the transport mechanism of an injection agent in building materials such as bricks and mortar served as a basis for the test procedure. It was gradually developed and constitutes an enhancement of the test procedure of the Dahlberg Institute, Wismar [5].

The transport of an injection agent - and also of water - is represented by the capillary pores of the building material (order of magnitude $10^{-3}$ to $10^{-7} \mathrm{~m}$ ). This means, the distribution and the frequency of the existing capillary pores in building materials are decisive for the transport of the injection agent and how extensively the existing pore spaces are already filled with water [6].

Consequently the following thesis resulted:

- with increased filling of the pore spaces with water, i.e. with increased moisture degree $D(g)$, less injection agent can be absorbed by the pore spaces; and

- a limited spread of the injection agent is to be expected.

\section{Materials and methods}

Two injection agents were chosen, both based on synthetic resin (here labeled as IM A and IM B, see Fig. 1). The physico-chemical properties as terms of density, 
reaction mechanism and viscosity were similar as well as the active principle of poreconstricting and hydrophobization. According to the manufacturers' specifications both injection agents are suitable for moisture levels up to $90 \%$. Due to the content of synthetic resin rubber of IM A in comparison to IM B, there is a crack-bridging effect of IM A.

The injection agents were applied by a pressure-less injection method, at different degrees of moisture penetration $D(g)$ as $0 \%, 50 \%, 75 \%, 90 \%$. That is to say, the application of the sealing substance was done by cartridges, partly with suction edges, while the intake was effected by means only of gravity and capillarity.

The building material chosen was solid bricks of common use of the order of 240x115x71 mm.

A test program was developed based on the Dahlberg Institute test procedure [5].

After application, the samples were split with a hammer and a smaller chisel (Fig. 2.) after making a small groove on the top of the building material with an angle grinder.

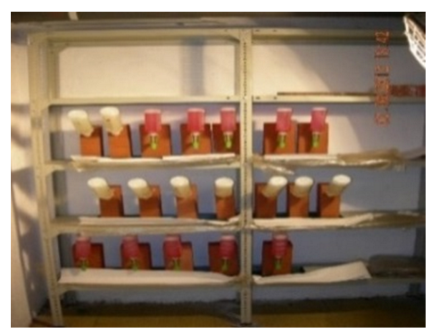

Fig. 1. Application of injection agents in bricks

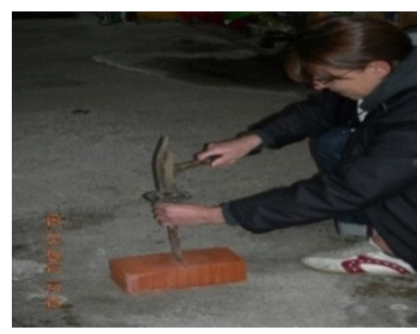

Fig. 2. Splitting of the samples

\section{Results}

Visual verification of the spread:

Frequently the spread of the injection agent can already be seen on the surface of the building material, recognizable by dark colored areas in the form of concentric diffusions around the borehole.

After splitting the samples it is also possible to recognize the spread at a greater depth. Additionally, it can already be seen, that the spread of an injection agent decreases with an increased degree of moisture penetration, as it is shown in the following figures (Fig. 3).

\section{Droplet test for the visual detection of the hydrophobic effect:}

If the surface of a building material is hydrophobic there is (mostly) no chance for water to be absorbed by capillary pores and to be transferred to greater depths within the material. 

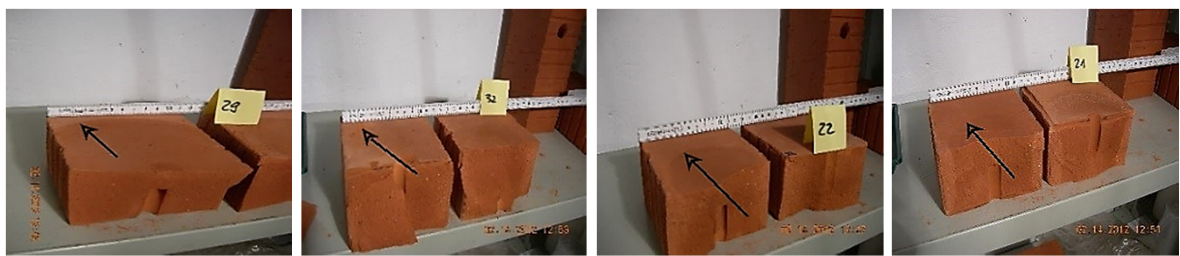

Fig. 3. Decreasing spread of the injection agent with increasing degree of moisture penetration (from left to right: degree of moisture penetration $D(g): 0 \%, 50 \%, 75 \%, 90 \%$ ), the limit of the spread is shown by the black arrow

The reason for this can be explained as follows: if a water droplet is set on the untreated surface of a building material, the edge angle of the droplet is less than 90 degrees and the droplet is soon passed to the inner pore structure by capillarity. In contrast, after hydrophobing the surface, the edge angle changes to above 90 degrees and the droplet remains on the surface of the building material (Fig. 4). This is the reason why hydrophobic injection agents are used for a horizontal sealing [7], [8], [9].

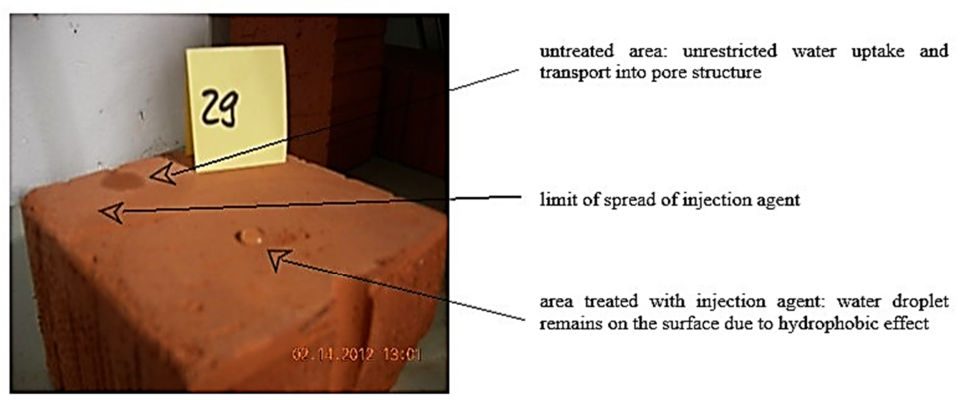

Fig. 4. Droplet test hydrophobic/non hydrophobic area

Representation of hydrophobicity by determining the water absorption coefficient, (w-value) according to EN ISO 15148

The amount of water uptake can be determined by the $w$-value on the basis of EN ISO 15148. Test specimens are exposed to water and the uptake of water is measured by weighting at regular intervals. The measured values can be displayed graphically as shown below in Fig. 5 and Fig. 6. As it can be seen, after application of an injection agent there is a significant effect on the $w$-value.

There is a diamond result (sample B* with $D(g) 75 \%$, red dots) in Fig. 6 while the other results are all below the value of $0.5 \mathrm{~kg} / \mathrm{m}^{2} \cdot \mathrm{h}^{0.5}$ and can therefore be classified as water-repellent [3].

On closer examination of the diamond result it could be seen that the test specimen was cut rectangular while the spreading was almost concentric. While performing the test procedure to determine the $w$-value, the water uptake was recognizable on the untreated areas (Fig. 7). 
This knowledge may allow the conclusion that the overlapping areas of the spreading radii must be correctly calculated in order to achieve a functional seal.

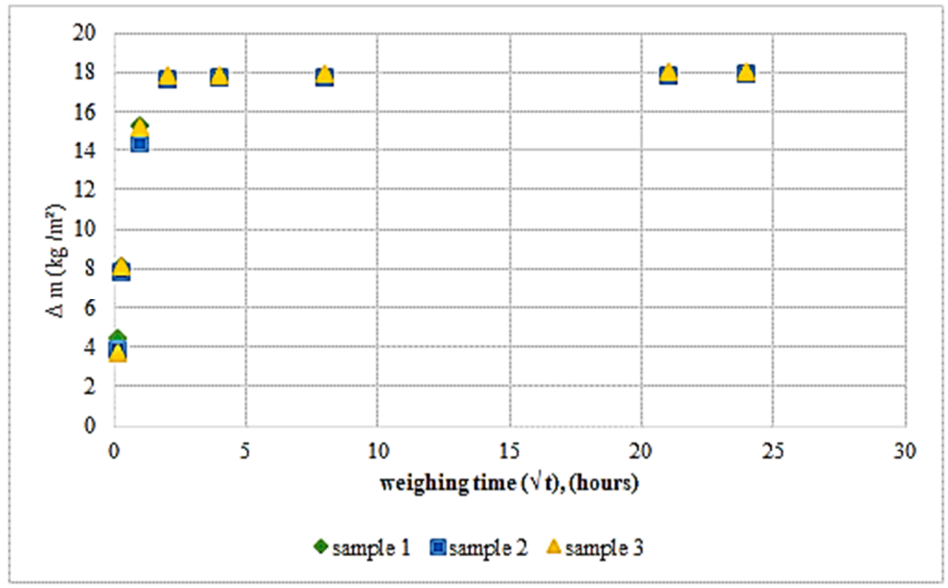

Fig. 5. w-value before application

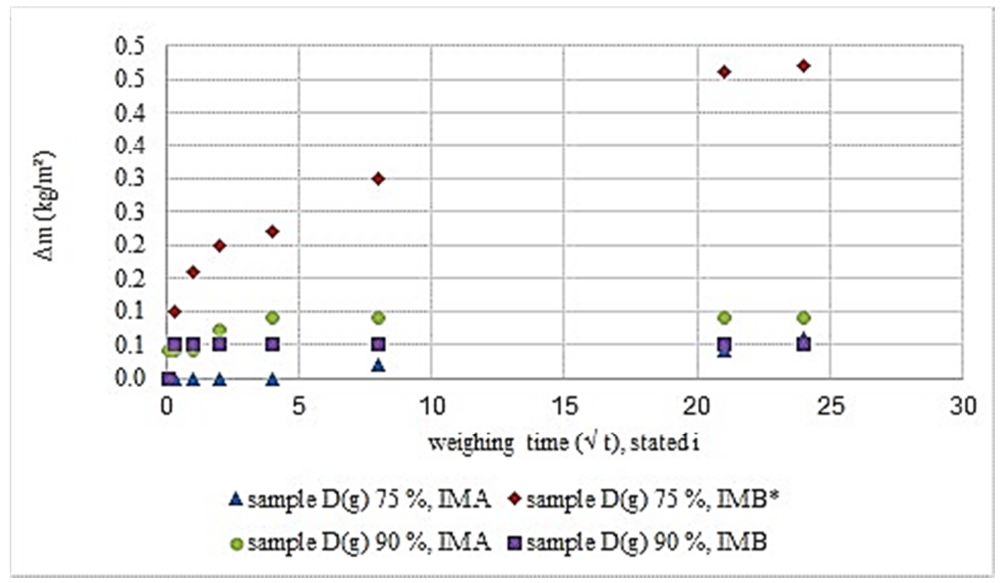

Fig. 6. w-value after application

Graphical presentation of the spread of the injection agent

Regarding the spread of an injection agent it is also possible to measure the distance of the spread from the borehole as shown in the next figure (Fig. 8):

Two important statements can be made:

- It is evident that the decrease of the spread of an injection agent is inversely proportional to the increase of moisture penetration;

- Though the injection agents are quite similar, the results may differ [6]. 

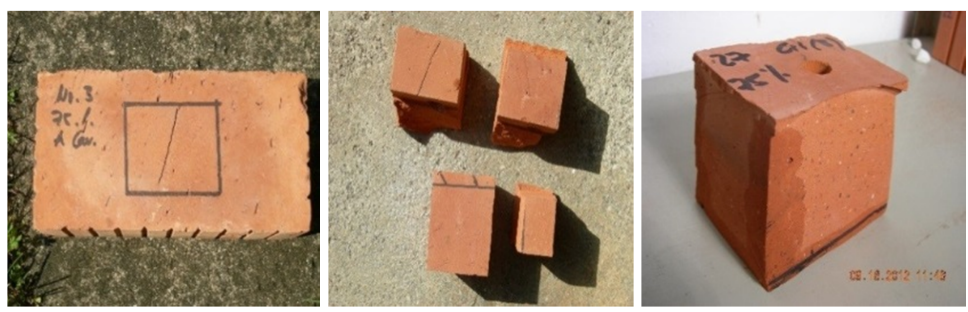

Fig. 7. Preparing the transition zone hydrophobic/non hydrophobic area of the test specimen to determine the $w$-value (left: marking of the dark colored hydrophobized area, middle: cut test specimen, right: unrestricted water absorption in the non-hydrophobic area)

\section{Spread of injection agent in relation to the degree of moisture penetration $\mathrm{D}(\mathrm{g})$}

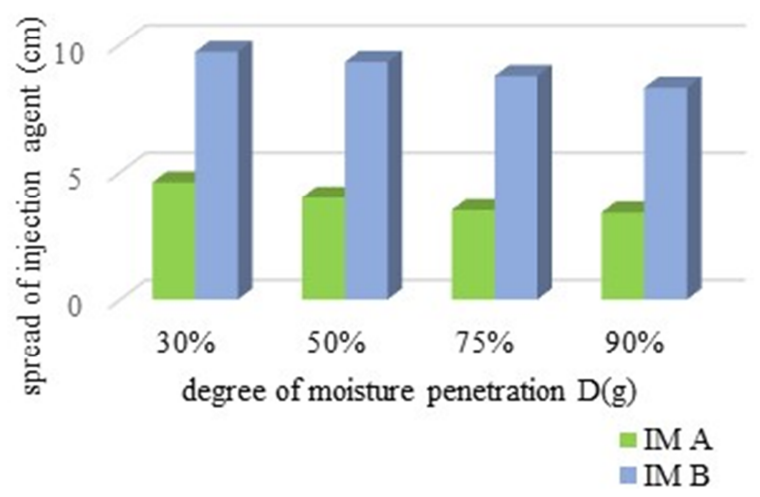

Fig. 8. Graphical presentation of the spread of two injection agents

Presentation of the spread of an injection agent using linear regression

Regression analyses are used as methods of statistical evaluation to determine relationships between a dependent and one or more independent variables.

On a first attempt the data was illustrated as follows (Fig. 9) and the following abbreviations are used in the figure: $\mathrm{x}$ is the existing degree of moisture penetration; $\mathrm{y}$ is the spread of injection agent, dependent on the existing degree of moisture penetration; $\mathrm{R}^{2}$ is the coefficient of determination as the square of the correlation coefficient.

In this connection the coefficient of determination as the square of the correlation coefficient is a first approximation, i.e. how much of the variance (the scattering of one variable) can be explained by the variance of the other variable and lies between 0 (no linear relationship) and 1 (linear relationship). As it can be seen in Fig. 9 the value of the coefficient of determination represents a linear relationship between the spread of an injection agent and the degree of moisture penetration. 


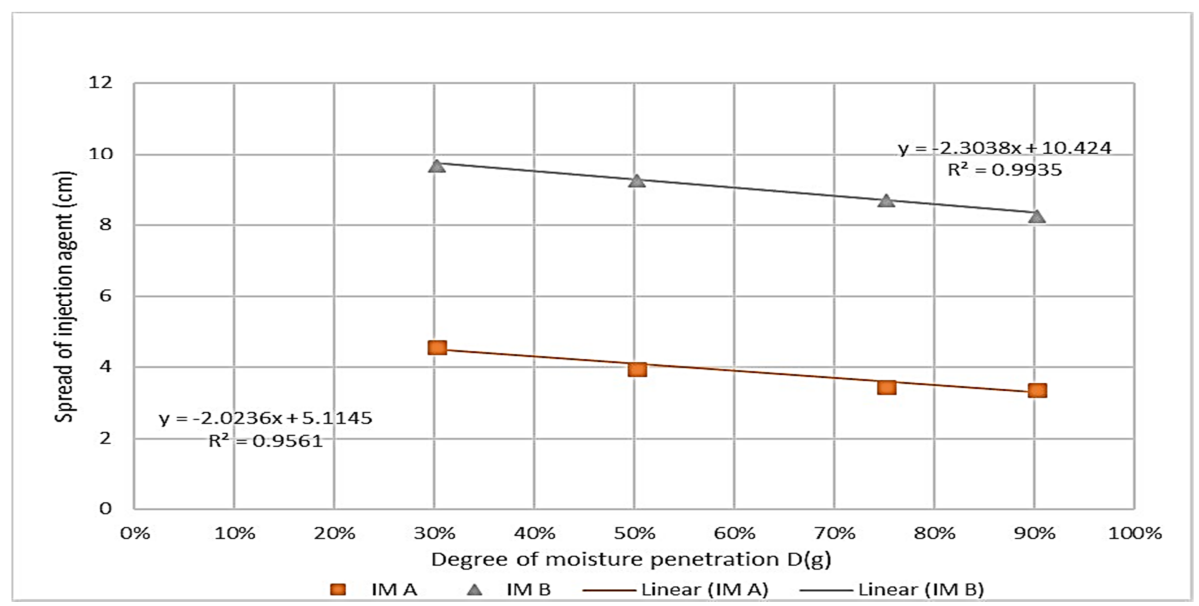

Fig. 9. Presentation of the spread using linear regression

\section{Modeling of the spread of an injection agent using linear regression}

As the above results showed a correlation of the investigated parameters, a model of the spread of an injection agent using linear regression was developed.

The expected result was the illustration of the dependence between the spread of an injection agent and the degree of moisture penetration $D(g)$.

A linear relationship is as follows:

$$
A_{D}=A_{\max }-A_{k}=A_{\max }-k_{i m} \cdot D(g),
$$

with the following abbreviations: $A$ is the spread of injection agent (in $\mathrm{cm}$ ) around the borehole; $D(g)$ is the degree of moisture penetration $(D(g))$; $A_{D}$ is the spread of injection agent at a certain degree of moisture penetration; $A_{\max }$ is the maximum spread at $D(g)=0 \% ; A_{k}$ is the correction variable (corresponds to the decreasing spread of the injection agent dependent on $D(g)$ - > the amount by which the maximum spread of an injection agent is reduced); $k_{i m}$ is the permeability of an injection agent (materialdependent, in cm pro $\% D(g))$, material parameter) $->$ describes the decrease of the moisture-dependent spread in relation to the increasing degree of moisture penetration $D(g)$.

The permeability $k_{i m}$ of an injection agent therefore describes its reduced spread (in $\mathrm{cm})$ when the degree of moisture penetration changes by $1 \%$.

This can be illustrated by measuring the degree of moisture penetration and the spread of the injection agent at two different locations on the building to be renovated (one location is preferably in the sealing horizon).

Then the following quotient can be calculated (values in $\mathrm{cm} / \%$ ):

$$
k_{i m}=\frac{A_{D_{1}}-A_{D_{2}}}{D_{1}-D_{2}} \text {, }
$$


where $A_{\max }$ is determined by an experiment, i.e. $D(g)$ is $0 \%$; that means, there is no moisture in the pore space, the material is 'dry'.

By substituting the parameters of the permeability and $A_{\max }$ in the equation (1), the characteristic curve can be presented graphically as it can be seen in Fig. 10.

$$
A_{D}=A_{\max }-A_{k}=A_{\max }-k_{i m} \cdot D
$$

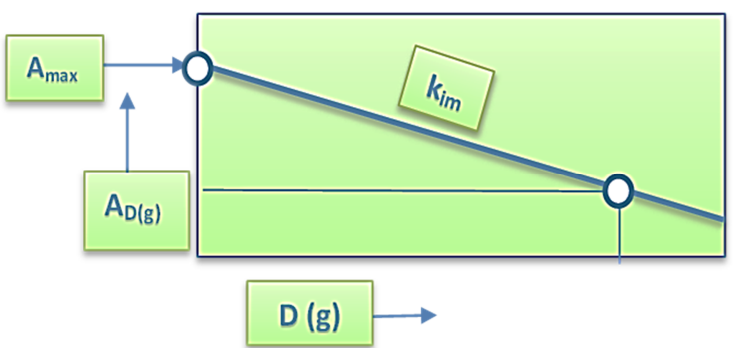

Fig. 10. Model of the spread of injection agent, $A_{D}$ is the spread of injection agent at a certain degree of moisture penetration; $A_{\max }$ is the maximum of spread at $D(g) 0 \% ; k_{i m}$ is the permeability of injection agent (material-dependent, in $\mathrm{cm}$ pro $\% D(g)$ ), material parameter); $D(g)$ is the degree of moisture penetration $D$

By using the two parameters in the above-mentioned equation (2) and use of values, a direct dependence of the moisture-induced spread of the injection agent $A_{D}$ and the degree of moisture penetration $D(g)$ results. $=>$ The characteristic curve represents the decrease of the spread of the injection agent with increasing $D(g)$.

Practical application of the modeling (here shown on bricks with injection agent based on synthetic resin)

With reference to the previously presented model of linear regression, the decrease of the spread of the injection agent can also be represented in terms of values (see Table I):

For example: At a degree of moisture penetration of $50 \%$ the 'correction' of the spreading is about $1.5 \mathrm{~cm}$. i.e. compared to the maximum spread of $5.5 \mathrm{~cm}$. the spread at $50 \% D(\mathrm{~g})$ is $5.5 \mathrm{~cm}-1.5 \mathrm{~cm}=4 \mathrm{~cm}$.

Spread of injection agents and distances of boreholes

The spread of an injection agent and the distances(s) between the necessary boreholes must be well matched, so the following information is required:

- Type of building material: bricks (e.g. bulk density), mortar, type of masonry composite (e.g. homogeneity, cavities);

- Type of injection agent;

- Characteristic values of existing degree of moisture penetration. 
Consequence: in order to create a functional sealing a continuous sealing level including overlapping areas of the spreading radii - is required to prevent further moisture intake.

\section{Table I}

Spread of injection agent dependent on the existing degree of moisture penetration

\begin{tabular}{|c|c|c|}
\hline$D(g)$ in \% & $A_{D}$ in cm & $\begin{array}{c}A_{k}=k_{\text {im }} D \\
\text { (in cm) }\end{array}$ \\
\hline 0 & $5.5\left(=A_{\max }\right)$ & -- \\
\hline 10 & 5.2 & 0.30 \\
20 & 4.9 & 0.60 \\
30 & 4.6 & 0.90 \\
40 & 4.4 & 1.1 \\
50 & 4.0 & 1.5 \\
60 & 3.8 & 1.68 \\
\hline 70 & 3.5 & 1.96 \\
\hline 80 & 3.4 & 2.08 \\
\hline 90 & 3.4 & 2.07 \\
\hline
\end{tabular}

The problem is as follows:

- the 'overlap' must be arranged in a way that rising moisture is avoided;

- the cost-effectiveness of the process must be considered (and possibly accompanying measures as e.g. convective pre-drying e.g.).

To start with the following suggestions have been made (draft, not true to scale) (see Fig. 11):

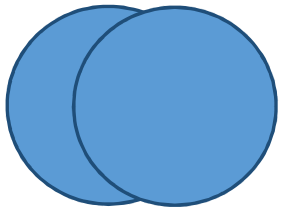

distance between the borehole is too small $\rightarrow$ not economic

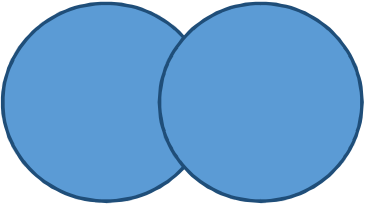

distance between the boreholes is well balanced (technical)

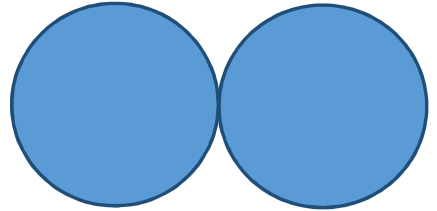

distance between the boreholes is too large $->$ possibly no functional sealing, risk of ascending moisture

Fig. 11. Suggestions reflection borehole distance

\section{Application of the modeling to calculate borehole distances}

The solution is to ensure the same overlapping areas at different degrees of moisture penetration. This can be illustrated by multiplication of the spread of an injection agent at a certain degree of moisture penetration $D(g)$ with a constant factor $f$ :

$$
B_{A}=f \cdots A_{D},
$$


where $B_{A}$ is the borehole distance. In order to ensure the same proportion of the overlapping areas of the spreading radii, a value range between 1.25 and 1.4 seems to be useful for factor $f$. The above mentioned values are the result on considerations on the economic efficiency and the technical functionality of the modeling.

Consequently the range of minimal borehole distance is

$$
B_{A, \min }=1.25 A_{D}=1.25\left(A_{\max }-k_{i m} \cdot D\right) \text { in } \mathrm{cm} \text {; }
$$

the range of maximal borehole distance is

$$
B_{A, \max }=1.4 A_{D}=1.4\left(A_{\max }-k_{i m} \cdot D\right) \text { in } \mathrm{cm}
$$

and therefore the determination of the range of borehole distances is

$$
B_{A, \min } \leq B_{A} \leq B_{A, \max }
$$

\section{Practical application of the model to calculate borehole distances}

As it can be seen in Table II, it is possible to determine the spread of an injection agent at certain degree of moisture penetration by means of the model. The distance between the boreholes at certain degrees of moisture penetration can also be calculated by means of our model.

\begin{tabular}{|c|c|c|c|c|}
\hline $\begin{array}{c}D(g) \text { in } \\
\%\end{array}$ & $A_{D}$ in $\mathrm{cm}$ & $\begin{array}{c}A_{k}=k_{\text {im }} D \\
\text { (in } \mathrm{cm} \text { ) }\end{array}$ & $\begin{array}{l}I M \text { permeability } \\
\text { (in } \mathrm{cm} / \% \text { ) }\end{array}$ & $\begin{array}{l}\text { Distance between boreholes } \\
\qquad B_{A}=1.4 A_{D}(\text { in } \mathrm{cm})\end{array}$ \\
\hline 0 & $5.5\left(=A_{\max }\right)$ & -- & -- & -- \\
\hline 10 & 5.2 & 0.30 & 0.030 & 7.2 \\
\hline 20 & 4.9 & 0.60 & 0.030 & 6.8 \\
\hline 30 & 4.6 & 0.90 & 0.030 & 6.4 \\
\hline 40 & 4.4 & 1.1 & 0.028 & 6.2 \\
\hline 50 & 4.0 & 1.5 & 0.030 & 5.6 \\
\hline 60 & 3.8 & 1.68 & 0.028 & 5.3 \\
\hline 70 & 3.5 & 1.96 & 0.028 & 4.9 \\
\hline 80 & 3.4 & 2.08 & 0.026 & 4.8 \\
\hline 90 & 3.4 & 2.07 & 0.023 & 4.8 \\
\hline
\end{tabular}

Table II

Application of the model to calculate borehole distances

For example: Regarding the above example and the 'corrected' spread of the injection agent of $4.0 \mathrm{~cm}$ at $50 \%$ degree of moisture penetration, the borehole distance should be $5.6 \mathrm{~cm}(4.0 \mathrm{~cm} \times \mathrm{f} 1.4)$. 


\section{Conclusions}

Main result:

The spread of an injection agent as well as the appropriate bore hole spacing, which is relevant for the functional capability of a subsequent horizontal sealing, can be represented not only visually but also by means of physical parameters.

\section{Further conclusions:}

- The spread of an injection agent is inversely proportional to the degree of moisture penetration $D(g)->$ the more moistened the masonry is, the smaller is the absorption of an injection agent (because of the filling of the capillary pores with water) and therefore the smaller the spread;

- In the range between $50 \%$ and $75 \% D(g)$, a marked decline of the injection agent is caused by an increased filling of the pore space with water. An injection at these degrees of moisture penetration is to be critically evaluated without accompanying measures;

- The degree of moisture penetration in masonry is generally to be taken into account when choosing the methods and/or the injection agent to be carried out and, if necessary, to be adapted (borehole spacing, pre-drying, etc.);

- Injection agents with similar chemical properties can spread differently in the same building materials $->$ the spread is determined not only by the building material, but also by the chosen injection agent;

- A well planned preliminary examination of the actual degree of moisture penetration and the type and condition of the building helps the planning of the renovation and contributes to the success of the subsequent horizontal seal;

- Consider also the qualification and the experience of the planning and execution staff;

- The spreading radii of IM must be overlap sufficiently; this can be achieved by adjusting the borehole distances;

- General information on borehole spacing, quantities of intake of injection agents after application etc. should be critically questioned

- The model presented has particular novelties regarding...

$\circ$ the definition of the terms as e.g. injection agent permeability, correction variable;

o the definition of the spread of injection agents at different degrees of moisture penetration;

○ the determination of the appropriate borehole spacing;

and can therefore make a valuable contribution to the effectiveness of a subsequent horizontal seal.

Further studies on the above mentioned topics (e.g. different types of mortar and varying bulk densities of bricks, several injection agents and the statistical evaluation) are still on-going and will be presented soon. 


\section{Acknowledgements}

The author would like to thank Prof. Dr. rer. nat. Dr. Ing. habil. Helmuth Venzmer as well as Prof. Dr. Ing. Winfried Malorny, Faculty of Engineering, University of Applied Sciences in Wismar/Germany, for the possibility of using the laboratory equipment.

\section{Open Access statement}

This is an open-access article distributed under the terms of the Creative Commons Attribution 4.0 International License (https://creativecommons.org/licenses/by/4.0/), which permits unrestricted use, distribution, and reproduction in any medium, provided the original author and source are credited, a link to the CC License is provided, and changes - if any - are indicated. (SID_1)

\section{References}

[1] Venzmer H., Walter A. Bautenschutz durch Abdichtung: Modellierung der Injektionsmittelsausbreitung in Mauerwerksbaustoffen, In: Venzmer H. (Ed.) Bautenschutz - Innovative Sanierungslösungen, Beuth Verlag GmbH, 2014, pp. 21-29.

[2] Walter A., Venzmer H. Nachweis von Injektionsmitteln in mineralischen Baustoffen mittels Thermischer Analyse (TA) In: Venzmer H. (Ed.) Energielieferant Altbau Potenziale, Nachhaltigkeit, Objektbeispiele, Beuth Verlag GmbH, 2017, pp. 77-84

[3] Kruis J., Koudelka T., Krejci T. Salt Transport in porous materials and its efficient computer implementation, Pollack Periodica, Vol. 9, No. 2, 2014, pp. 111-122.

[4] Krejci T., Koudelka T., Kruis J. Numerical modeling of coupled hydrothermo-mechanical behavior of concrete structures, Pollack Periodica, Vol. 10, No. 1, pp. 19-30.

[5] Dahlberg Institut für Diagnostik und Instandsetzung historischer e.V. Bausubstanz, Wismar am sanierten Bauwerk durch die Kenngröße Abdichtungsqualität AQ, Beuth Publishing Company, 2012.

[6] Wojcik R., Panus A., Tunkiewicz M., Hamdy M. Influence of chemical damp proof cream on the capillary action and microstructure of mortars, $11^{\text {th }}$ Nordic Symposium on Building Physics, NSB2017, Trondheim, Norway, 11-14 June 2017, Energy Procedia, Vol. 132, 2017, pp. 670-675.

[7] Hansen T., Bjarlov S., Peuhkuri R. Moisture transport properties of brick - comparison of exposed, impregnated and rendered brick, International RILEM Conference on Materials, Systems and Structures in Civil Engineering, segment on Moisture in Materials and Structures, Trondheim, Norway, 11-14 June 2016, pp. 351-360.

[8] Hacquebord A., Lubelli B., van Hees R., Nujland T. Evaluation of spreading and effectiveness of injection products against rising damp on mortar/brick combinations, Procedia Chemistry, Vol. 8, 2013, pp. 139-149.

[9] Fic S., Barnat-Hunek D. The effectiveness of hydrophobisation of porous building materials by using the polymers and nanoploymers solutions, International Journal of Materials Science and Engineering, Vol. 2, No. 2, 2014, pp. 93-98 